\title{
POSITIVE SEMIDEFINITE FORMS OVER ORDERED SKEW FIELDS
}

\author{
KA HIN LEUNG
}

(Communicated by Donald S. Passman)

\begin{abstract}
In any ordered field $K$, the inequality $x^{2}+y^{2} \geq 2 x y$ obviously holds for any $x, y \in K$. Naturally, we may ask if the same inequality holds in every ordered skew field. Surprisingly, it can be proved that in an ordered domain $R$, the above inequality holds for any elements $x, y$ in $R$ iff $R$ is commutative. In this paper, we formulate a generalization of the above observation and prove that if a "positive semidefinite form" over an ordered skew field admits a "nontrivial" solution, then the skew field is actually a field.
\end{abstract}

\section{FORMS OVER SKEW FIELDS}

The notion of positive semidefinite (P.S.D.) polynomials has been known for a long time. In fact, the famous Hilbert's Problem 17 asked if every positive semidefinite polynomial $f \in k\left[x_{1}, \ldots, x_{n}\right]$ can be expressed as a sum of squares in $k\left(x_{1}, \ldots, x_{n}\right)$, where $k$ is either $\mathbf{Q}$ or $\mathbf{R}$. For a survey on this problem, we refer the readers to [L, §7]. In the 1970s, people became interested in studying positive semidefinite forms (over $\mathbf{R}$ ) instead of polynomials. They studied the cones of P.S.D. forms and S.O.S. forms (i.e. forms which can be expressed as sums of squares of other forms) of some fixed degree. For details, we refer the readers to $[R],\left[\mathrm{CL}_{1}\right],\left[\mathrm{CL}_{2}\right],\left[\mathrm{CLR}_{1}\right],\left[C L R_{2}\right]$ and $\left[\mathrm{CLR}_{3}\right]$. Nevertheless, the notion of positive semidefinite forms over an ordered skew field does not seem to have received any attention. Firstly, let us define the notion of forms over a skew field.

Definition 1.1. Let $D$ be a skew field, and $x_{1}, \ldots, x_{n}$ be noncommuting indeterminates. We say $F\left(x_{1}, \ldots, x_{n}\right) \in D\left[x_{1}, \ldots, x_{n}\right]$ is a form of degree $2 N$ if

$$
F\left(x_{1}, \ldots, x_{n}\right)=\sum_{i_{1}+\cdots+i_{n}=2 N} a_{\left(i_{1}, \ldots, i_{n}\right)} x_{1}^{i_{1}} \cdots x_{n}^{i_{n}},
$$

Received by the editors October 14, 1988 and, in revised form, January 13, 1989.

1980 Mathematics Subject Classification (1985 Revision). Primary 16A86; Secondary 16A39, $16 \mathrm{~A} 70$. 
for some $a_{\left(i_{1}, \ldots, i_{n}\right)} \in D$, and not all $a_{i}$ 's are zero. Furthermore, for any $y_{1}, \ldots, y_{n} \in D$ we define:

$$
F\left(y_{1}, \ldots, y_{n}\right):=\sum_{i_{1}+\cdots+i_{n}=2 N} a_{\left(i_{1}, \ldots, i_{n}\right)} y_{1}^{i_{1}} \cdots y_{n}^{i_{n}} .
$$

Remark. Note that according to our definition, $x_{1}^{2}-x_{1} x_{2}-x_{2} x_{1}+x_{2}^{2}$ is not a form.

Definition 1.2. Let $R$ be a ring. We say $P \subset R$ is an ordering on $R$ if (i) $P+P \subset P$, (ii) $P \cdot P \subset P$, (iii) $P \cap-P=\{0\}$, (iv) $P \cup-P=R$. In this case, we say $(R, P)$ is an ordered ring and write $a>0$ if $a \in P \backslash\{0\}$.

Definition 1.3. Let $D$ be an ordered skew field. We call a form $F\left(x_{1}, \ldots, x_{n}\right) \in$ $D\left[x_{1}, \ldots, x_{n}\right]$ positive semidefinite (P.S.D.) if

$$
F\left(y_{1}, \ldots, y_{n}\right) \geq 0 \quad \forall y_{1}, \ldots, y_{n} \in D \text {. }
$$

Examples of P.S.D. forms are abundant. Forms such as $F_{1}\left(x_{1}, x_{2}\right):=x_{1}^{2}+x_{2}^{2}$, $F_{2}\left(x_{1}, x_{2}\right):=x_{1}^{2} x_{2}^{2}+x_{2}^{4}$ and $F_{3}\left(x_{1}, x_{2}\right):=x_{1}^{2}-x_{1} x_{2}+x_{2}^{2}$ are P.S.D. over any ordered skew field. To see that $F_{3}$ is P.S.D., just observe that if $x y<0$, then obviously $F_{3}(x, y)>0$. If $x y>0$, then also $y x>0$ and $F_{3}(x, y)=$ $(x-y)^{2}+y x>0$. Let us consider another form $F_{4}\left(x_{1}, x_{2}\right):=x_{1}^{2}+2 x_{1} x_{2}+x_{2}^{2}$. As we have pointed out earlier, $F_{4}$ is P.S.D. over any ordered field, but fails to be P.S.D. over any noncommutative ordered skew field. To distinguish these forms, we introduce the following definition.

Definition 1.4. Let $F\left(x_{1}, \ldots, x_{n}\right)$ be a form over a skew field $D$. We say $F$ admits a nontrivial solution if there exists $\left(y_{1}, \ldots, y_{n}\right) \in\left(D^{*}\right)^{n}$ such that $F\left(y_{1}, \ldots, y_{n}\right)=0$.

Remark. Later one we shall relax the definition of nontrivial solution.

Observe that $F_{1}, F_{2}, F_{3}$ have no nontrivial solution whereas $F_{4}$ admits nontrivial solutions. As we shall show later, for any P.S.D. form $F$ over an ordered skew field $D$, if $F$ admits a nontrivial solution, $D$ must be a field. In particular, this explains why $x_{1}^{2}-2 x_{1} x_{2}+x_{2}^{2}$ fails to be P.S.D. over any noncommutative ordered skew field. This also explains why we do not allow $x_{1}^{2}-x_{1} x_{2}-x_{2} x_{1}+x_{2}^{2}$ to be a form, as it is clearly positive semidefinite and has infinitely many nontrivial solutions.

\section{ON THE HOLDING OF CERTAIN INEQUALITIES}

From now on, let us fix an ordered skew field $D$ and an indeterminate $t$. We denote the coproduct of $D$ and $Z(D)[t]$ by $D\langle t\rangle$ (i.e., $D\langle t\rangle$ is a ring generated by $D$ and $t$ with $t$ commuting with elements in $Z(D)$ only). Furthermore, we define the following:

$$
\mathscr{P}:=\left\{a_{1} t a_{2} t \cdots a_{n} t a_{n+1} \in D\langle t\rangle: n \geq 2, a_{i} \in D^{*}\right\},
$$


and

$$
D[t]:=\left\{\sum_{i=0}^{n} a_{i} t^{i} \in D\langle t\rangle: a_{i} \in D\right\} .
$$

Here, our main goal is to show that if $f(t) \in D[t]$ is positive semidefinite (i.e. $\forall x \in D, f(x) \geq 0$ ), then $f(y)=0$ implies $y \in Z(D)$. This result turns out to be crucial in studying P.S.D. forms admitting a nontrivial solution.

Lemma 2.1. Let $f(t)=\sum_{i=r}^{s} a_{i} t^{i} \in D[t]$ be a P.S.D. polynomial with both $a_{r}$, $a_{s}$ being nonzero. Then $r, s$ are even, and $a_{r}, a_{s}>0$.

Proof. It is not difficult to see that if $|x|$ is sufficiently big, then $a_{s} x^{s}$ will dominate all other terms. Thus $f(x) \geq 0$ implies $s$ is even and $a_{s}>0$. Similarly, if $|x|$ is sufficiently small, then $a_{r} x^{r}$ will dominate. Thus again, $r$ must be even and $a_{r}>0$.

Next, for any $a_{1}, \ldots, a_{n}, b_{1}, \ldots, b_{n} \in D^{*}, p_{1}(t), \ldots, p_{m}(t) \in \mathscr{P}$, we want to study the conditions under which

$$
\left|\sum_{i=1}^{a} a_{i} x b_{i}\right| \leq \sum_{j=1}^{m}\left|p_{j}(x)\right|
$$

holds for any $x \in D$.

Lemma 2.2. For any $p_{1}(t), \ldots, p_{m}(t) \in \mathscr{P}$, there exists positive $\delta \in D^{*}$ such that $0<|x|<\delta$ implies $|x|>\sum_{i=1}^{m}\left|p_{i}(x)\right|$.

Proof. Let $p(t):=a_{1} t a_{2} t \cdots t a_{n+1} \in \mathscr{P}$. Observe that by definition of $\mathscr{P}$, $n \geq 2$. For any $|x|<1$ in $D$, we have

$$
|p(x)| \leq\left|a_{1} \cdots a_{n-1} x a_{n} x a_{n+1}\right| .
$$

Thus if we take $b=\max \left\{\left|a_{1} \cdots a_{n-1}\right|,\left|a_{n}\right|,\left|a_{n+1}\right|\right\}$, then $|p(x)| \leq|b x b x b|$ $\forall|x|<1$. Consequently, there exists $b_{j} \in D$ such that $\left|p_{j}(x)\right| \leq\left|b_{j} x b_{j} x b_{j}\right|$ whenever $|x|<1$. If we let $a:=m \cdot \max _{1 \leq j \leq m} b_{j}$, then we have

$$
\sum_{j=1}^{m}\left|p_{j}(x)\right| \leq \mid \text { axaxa }|\quad \forall| x \mid<1 .
$$

Therefore to prove the lemma, it suffices to show that for any $a \in D^{*}$, there exists positive $\delta \in D$ such that whenever $0<|x|<\delta$, we have $|x|>\mid$ axaxa $\mid$.

Take any $\delta \in D^{*}$ such that $0<\delta<\min \left\{1,|a|^{-3}\right\}$, then obviously $|x|<\delta$ implies $|a x a|<|a|^{-1}$. Therefore,

$$
\mid \text { axaxa }\left|<\min \left\{\left|a^{-1} x a\right|,\left|a x a^{-1}\right|\right\} \leq\right| x \mid \text {. }
$$

Proposition 2.3. Let $n \in \mathbf{N}, a_{1}, \ldots, a_{n}, b_{1}, \ldots, b_{n} \in D^{*}$ and $p_{1}(t), \ldots$, $p_{m}(t) \in \mathscr{P}$. If

$$
\left|\sum_{i=1}^{n} a_{i} x b_{i}\right| \leq \sum_{j=1}^{m}\left|p_{j}(x)\right| \quad \forall x \in D,
$$


then $n \geq 2$ and $\sum_{i=1}^{n} a_{i} t b_{i}=0$ in $D\langle t\rangle$. In particular, $\left\{a_{1}, \ldots, a_{n}\right\},\left\{b_{1}, \ldots\right.$, $\left.b_{n}\right\}$ are linearly dependent over $Z(D)$.

Proof. Obviously, we may assume $a_{1}=b_{1}=1$. If $n=1$, then it contradicts Lemma 2.2. Hence $n \geq 2$.

Let $n \geq 2$ be the smallest integer such that there exist $a_{1}, \ldots, a_{n}, b_{1}, \ldots$, $b_{n} \in D^{*}, p_{1}(t), \ldots, p_{m}(t) \in \mathscr{P}$ with $\sum_{i=1}^{n} a_{i} t b_{i} \neq 0$ in $D\langle t\rangle$ and such that inequality (1) holds. Clearly, $\left\{a_{1}, \ldots, a_{n}\right\},\left\{b_{1}, \ldots, b_{n}\right\}$ are then linearly independent over the center. As before, we assume $a_{1}=b_{1}=1$. By assumption, we have

$$
\left|x+\sum_{i=2}^{n} a_{i} x b_{i}\right| \leq \sum_{i=1}^{m}\left|p_{i}(x)\right| \quad \forall x \in D .
$$

For any $c \in D^{*}$, we replace $x$ by $c x$ and get

$$
\left|c x+\sum_{i=2}^{n} a_{i} c x b_{i}\right| \leq \sum_{i=1}^{m}\left|p_{i}(c x)\right| \quad \forall x \in D .
$$

Multiplying both sides by $|c|^{-1}$, we get

$$
\left|x+\sum_{i=2}^{n} c^{-1} a_{i} c x b_{i}\right| \leq \sum_{i=1}^{m}\left|c^{-1} p_{i}(c x)\right| \quad \forall x \in D .
$$

Combining inequalities (2) and (3), we conclude that

$$
\left|\sum_{i=2}^{n}\left(c^{-1} a_{i} c-a_{i}\right) x b_{i}\right| \leq \sum_{i=1}^{m}\left(\left|p_{i}(x)\right|+\left|c^{-1} p_{i}(c x)\right|\right) \quad \forall x \in D .
$$

Since $\left\{b_{1}, \ldots, b_{n}\right\}$ is linear independent over the center, it follows by assumption on $n$ that $c^{-1} a_{i} c-a_{i}=0$ for $2 \leq i \leq n$. Since $c$ is chosen arbitrarily, we conclude that all $a_{i}$ 's are central, and hence $\left\{a_{1}, \ldots, a_{n}\right\}$ is linearly dependent over the center. This is a contradiction.

Corollary 2.4. Let $0 \neq g(t)=\sum_{i=0}^{2 n} a_{i} t^{i}$ be a P.S.D. polynomials in $D[t]$. If $g(y)=0$ for some $y \in D^{*}$, then $y$ is central.

Proof. Since $g(t) \neq 0$, we may assume $a_{2 n} \neq 0$ by Lemma 2.1. For all $s \in D$,

$$
\begin{aligned}
g(y+s) & =\sum_{i=0}^{2 n} a_{i}(y+s)^{i} \\
& =g(y)+a_{1} s+\sum_{i=2}^{2 n} a_{i}\left(y^{i-1} s+y^{i-2} s y+\cdots+s y^{i-1}\right)+\sum_{j=1}^{k} p_{j}(s) \\
& =\sum_{i=0}^{2 n-1}\left(\sum_{j=i+1}^{2 n} a_{j} y^{j-i-1}\right) s y^{i}+\sum_{j=1}^{k} p_{j}(s) \geq 0
\end{aligned}
$$


for some $p_{j} \in \mathscr{P}$. Replacing $s$ by $-s$ if necessary, we may assume

$$
\sum_{i=0}^{2 n-1}\left(\sum_{j=i+1}^{2 n} a_{j} y^{j-i-1}\right) s y^{i} \leq 0 .
$$

Therefore, we have

$$
\sum_{j=1}^{k}\left|p_{j}(s)\right| \geq\left|\sum_{i=0}^{2 n-1}\left(\sum_{j=i+1}^{2 n} a_{j} y^{j-i-1}\right) s y^{i}\right| \quad \forall s \in D .
$$

As both $a_{2 n}$ and $y$ are nonzero, $\sum_{j=i+1}^{2 n} a_{j} y^{j-i-1} \neq 0$ for some $0 \leq i \leq 2 n-1$. (Actually, it is clear that the summand is nonzero for $i=2 n-1$ ). After dropping those zero terms, we can apply Proposition 2.3 to conclude that for some $i_{j} \in \mathbf{N},\left\{y^{i_{1}}, y^{i_{2}}, \ldots, y^{2 n-1}\right\}$ is linearly dependent over the center. Thus by Albert's theorem [A], $y$ is central.

\section{MAIN ResUlt}

In this section, we shall prove that if $D$ is not a field, then no P.S.D. form has a nontrivial solution. In the following, we always assume $F\left(x_{1}, \ldots, x_{n}\right)$ is P.S.D. of degree $2 N$ over the ordered skew field $D$.

Lemma 3.1. Let $\left(y_{1}, \ldots, y_{n}\right) \in\left(D^{*}\right)^{n}$ be a nontrivial solution of $F$ with $y_{r+1}$, $\ldots, y_{n}$ being central for some $r \leq n$ (where for $r=n$, the condition is vacuous). Moreover we define $g_{r}(t):=F\left(y_{1}, \ldots, y_{r-1}, t, y_{r+1}, \ldots, y_{n}\right)$.

(i) If $g_{r}(t) \neq 0$ in $D\langle t\rangle$, then $y_{r}$ is central.

(ii) If $g_{r}(t)=0$, then $\left(y_{1}, \ldots, y_{r-1}, 1, y_{r+1}, \ldots, y_{n}\right)$ is another nontrivial solution of $F$.

Proof. Since $y_{r+1}, \ldots, y_{n}$ are central, after moving them to the left side of $t$, we see that $g_{r}(t) \in D[t]$. As $F$ is P.S.D., $g_{r}(t)$ is also P.S.D.

Case (i). $g_{r}(t) \neq 0$. Since $g_{r}\left(y_{r}\right)=0$, by Corollary $2.4 y_{r}$ is central.

Case (ii). $g_{r}(t)=0$. Obviously, we have $g_{r}(1)=F\left(y_{1}, \ldots, y_{r-1}, 1, y_{r+1}\right.$, $\left.\ldots, y_{n}\right)=0$.

Corollary 3.2. Let $\left(y_{1}, \ldots, y_{n}\right) \in(Z(D))^{n}$ be a nontrivial solution of $F$ and $g_{n}(t):=F\left(y_{1}, \ldots, y_{n-1}, t\right)$. If $g_{n}(t) \neq 0$ in $D[t]$, then $D$ is a field.

Proof. Let $\left(y_{1}, \ldots, y_{n}\right)$ be a solution as assumed above. Since $F$ is homogeneous, and $y_{1}, \ldots, y_{n}$ are central, it follows that for any $x \in D^{*},\left(y_{1} x, \ldots\right.$, $\left.y_{n} x\right)$ is also a nontrivial solution of $F$. Moreover if $g_{n}(t)=\sum_{r}^{s} b_{i} t^{i} \neq 0$ in $D[t]$, then $h_{n}(t)=F\left(y_{1} x, \ldots, y_{n-1} x, t\right)=\sum_{i=r}^{s} b_{i} x^{2 N-i} t^{i} \neq 0$ in $D[t]$, where $2 N=\operatorname{deg} F$. Thus by Lemma 3.1, $y_{n} x$ is central. This clearly implies $x$ is central. Since this is true for all $x \in D^{*}, D$ is a field.

Theorem 3.3. Let $F\left(x_{1}, \ldots, x_{n}\right) \neq 0$ be a P.S.D. form of degree $2 N$ over $D$. If there exists a nontrivial solution $\left(y_{1}, \ldots, y_{n}\right)$, then $D$ is a field.

Proof. Claim. $y_{1}, \ldots, y_{n}$ can be assumed central. 
By Lemma 3.1, we see that either $y_{n}$ is central or $\left(y_{1}, \ldots, y_{n-1}, 1\right)$ is another nontrivial solution of $F$. Replacing $y_{n}$ by 1 if necessary, we can assume $y_{n}$ is central. Thus, by applying Lemma 3.1 repeatedly (replacing $y_{r}$ with 1 if necessary), the desired claim follows.

Next, we shall prove the theorem by induction on the number of variables $n$. If $n=2$, then $F\left(x_{1}, x_{2}\right)=\sum_{i=r}^{s} a_{i} x_{1}^{2 N-i} x_{2}^{i}$. Since $F \neq 0$, we can assume $a_{s} \neq 0$. Let $\left(y_{1}, y_{2}\right)$ be a nontrivial solution in the center and $g_{2}(t):=$ $\sum_{i=r}^{s} a_{i} y_{1}^{2 N-i} t^{i}$. As $y_{1} \neq 0$ and $a_{s} \neq 0$, it follows that $g_{2}(t) \neq 0$ in $D[t]$. Therefore, by Corollary 3.2, $D$ is a field.

Let $n \geq 2$ and $F\left(x_{1}, \ldots, x_{n+1}\right)=\sum_{i=r}^{s} F_{i}\left(x_{1}, \ldots, x_{n}\right) x_{n+1}^{i}$ be a P.S.D. form with nontrivial solution $\left(y_{1}, \ldots, y_{n+1}\right) \in\left(Z(D)^{*}\right)^{n+1}$. Consider

$$
g_{n+1}(t):=F\left(y_{1}, \ldots, y_{n}, t\right)=\sum_{i=r}^{s} F_{i}\left(y_{1}, \ldots, y_{n}\right) t^{i} .
$$

Case (i). $g_{n+1}(t) \neq 0$ in $D[t]$. Since $y_{1}, \ldots, y_{n+1}$ are central, by Corollary 3.2 , it follows that $D$ is a field.

Case (ii). $g_{n+1}(t)=0$ in $D[t]$. In this case, we have $F_{r}\left(y_{1}, \ldots, y_{n}\right)=0$. Observe that $r \neq 2 N$, otherwise $F\left(x_{1}, \ldots, x_{n+1}\right)=a x_{n+1}^{2 N}$ for some $a \in D^{*}$, and $F$ has no nontrivial solution. Therefore, $F_{r}$ is not a constant and we can regard it as a form with $n$ variables. Now clearly $g(t):=F\left(a_{1}, \ldots, a_{n}, t\right)=$ $\sum_{i=r}^{s} F_{i}\left(a_{1}, \ldots, a_{n}\right) t^{i} \in D[t]$ is P.S.D. for any $\left(a_{1}, \ldots, a_{n}\right) \in\left(D^{*}\right)^{n}$. By Lemma $2.1, F_{r}\left(a_{1}, \ldots, a_{n}\right) \geq 0$ for any $\left(a_{1}, \ldots, a_{n}\right) \in\left(D^{*}\right)^{n}$. Therefore $F_{r}$ is P.S.D. So by induction, $D$ is a field.

We are now ready to relax the definition of nontrivial solutions. Let $F\left(x_{1}\right.$, $\left.\ldots, x_{n}\right)$ be a form and $\left(y_{1}, \ldots, y_{n}\right) \in D^{n}$ be a solution of $F$. For $i=$ $1, \ldots, n$, we define $t_{i}$ to be $x_{i}$ if $y_{i} \neq 0$, otherwise, we define $t_{i}:=0$. Clearly, $F\left(t_{1}, \ldots, t_{n}\right) \in D\left[x_{1}, \ldots, x_{n}\right]$ is a form with number of variables less than or equal to $n$.

Definition 3.4. We say $\left(y_{1}, \ldots, y_{n}\right)$ is a nontrivial solution of $F$, if $F\left(y_{1}\right.$, $\left.\ldots, y_{n}\right)=0$ and $F\left(t_{1}, \ldots, t_{n}\right) \neq 0$ in $D\left[x_{1}, \ldots, x_{n}\right]$.

Let $F\left(x_{1}, \ldots, x_{n}\right)$ be a P.S.D. form with a nontrivial solution $\left(y_{1}, \ldots, y_{n}\right)$ (in the sense of Definition 3.4). Then the corresponding form $F\left(t_{1}, \ldots, t_{n}\right)$ will also be P.S.D. Moreover, if we drop all those zero-entries in $\left(y_{1}, \ldots, y_{n}\right)$, we get a nontrivial solution (in the sense of Definition 1.4) for the form $F\left(t_{1}\right.$, $\left.\ldots, t_{n}\right)$. Thus, by Theorem 3.3 , we conclude that $D$ is a field. Therefore, Theorem 3.3 is still true if we define the notion of nontrivial solution in the sense of Definition 3.4.

\section{FORMS OVER ORDERED RINGS}

After treating forms over ordered division rings, we attempt to generalize the above theorem to any ordered ring. Unfortunately, as we show below, Theorem 3.3 is no longer true over some ordered domains. 
Example. Let $\sigma$ be an automorphism on $\mathbf{Q}[a]$ sending $a$ to $2 a$ and $R:=$ $\mathbf{Q}[a][b ; \sigma]$ be the skew polynomial ring in $b$, i.e. $a b=2 b a$ and $a^{i} b^{j}=$ $2^{i j} b^{j} a^{i}$. Any $f \in R$ is of the form $f=\sum_{i=0}^{n} f_{i}(a) b^{i}$, such that $f_{i}(a)=$ $\sum_{j=1}^{l(i)} c_{i j} a^{j}, c_{i j} \in \mathbf{Q}$ and $c_{n l(n)} \neq 0$. Then $R$ is totally ordered by defining $0<f \in R$ if and only if $0<c_{n l(n)} \in \mathbf{Q}$.

Consider now the form $F\left(x_{1}, x_{2}\right)=a^{2} x_{1}^{2}-2 a x_{1} x_{2}+x_{2}^{2}$. Clearly we have $F(1, a)=0$. Next, we show $F$ is actually P.S.D.

For any $g=c a^{p} b^{n}+$ l.o.t.,$h=d a^{q} b^{m}+$ l. o. t. $\in R \backslash\{0\}$, with $0 \neq c, 0 \neq$ $d \in \mathbf{Q}$, and where "l.o.t." denotes lower order terms in $b$. It is straightforward to check that

$$
\begin{gathered}
a^{2} g^{2}=c^{2}(1 / 2)^{n p} a^{2 p+2} b^{2 n}+\text { l. o. t. } \\
2 a g h=2 c d(1 / 2)^{n q} a^{p+q+1} b^{n+m}+\text { l.o.t. } \\
h^{2}=d^{2}(1 / 2)^{m q} a^{2 q} b^{2 m}+1 . \text { o.t. }
\end{gathered}
$$

Case (i). $n \neq m$ or $q \neq p+1$. In this case, $F(g, h)=(1 / 2)^{n p} c^{2}+1$.o.t. or $(1 / 2)^{m q} d^{2}+1$.o.t. Since both are positive, we have $F(g, h)>0$.

Case (ii). $n=m$ and $q=p+1$. Thus $F(g, h)=(1 / 2)^{n p+n}\left(2^{n} c^{2}-2 c d+\right.$ $\left.d^{2}\right)+$ l.o.t. , if the later expression is nonzero. In fact, it is easy to check that $\left(2^{n} c^{2}-2 c d+d^{2}\right)>0$ if $n \neq 0$. In case $n=0, F(g, h)=(a g-h)^{2} \geq 0$.

Hence, $F$ is P.S.D. But by construction of $R, R$ is noncommutative.

Remark. As pointed out by the referee, $R$ can be embedded in the totally ordered formal skew power series division ring $D$ in power of $b$. Then the form $F\left(x_{1}, x_{2}\right)$ is not P.S.D. on $D$, as is required by Theorem 3.3.

Though we cannot generalize the main theorem to the case of ordered domains, we do have a similar result under certain circumstances.

Proposition 4.1. Let $(R, P)$ be an ordered domain. If for any $x, y \in R$, there exists $m(x, y) \in \mathbf{N}$ such that

$$
|x y-y x| \leq m(x, y) \min \left\{x^{2}, y^{2}\right\},
$$

then $R$ is commutative.

Proof. Firstly, we shall assume $R$ contains an identity. Let

$$
\begin{aligned}
& A=\{a \in R: n|a|<1 \forall n \in \mathbf{N}\}, \\
& B=\{a \in R: 1<n|a|<m \text { for some } n, m \in \mathbf{N}\}, \\
& C=\{a \in R: n<|a| \forall n \in \mathbf{N}\} .
\end{aligned}
$$

Clearly $A \cup B \cup C=R$. Observe that if $A=C=\varnothing$, then $R$ is archimedean. By [F, Chapter VIII, Theorem 1], we see that $(R, P)$ is order-isomorphic to a subring of $\mathbf{R}$. Thus $R$ is commutative. So we can assume $A$ or $C \neq \varnothing$.

Claim 1. If $A \neq \varnothing$, then any $x \in A \cup B$ is central. 
Let $x \in A \cup B$. For any $y \in R, a \in A \backslash\{0\}$, we have

$$
\begin{aligned}
\left|a^{4}(x y-y x)\right| & \leq\left|a^{4} x y-y a^{4} x\right|+\left|y a^{4} x-a^{4} y x\right| \\
& \leq n\left|a^{4} x\right|^{2}+m\left|a^{4}\right|^{2}|x| \quad \text { for some } n, m \in \mathbf{N} .
\end{aligned}
$$

As $R$ is a domain, after cancelling $\left|a^{4}\right|$, we get

$$
|x y-y x| \leq n\left|x a^{4} x\right|+m\left|a^{4} x\right| \text {. }
$$

Since $x$ is bounded by some integer $M$, and $n|x a|, m|a|$ are all $<1$. It follows that $|x y-y x| \leq|a| \forall a \in A \backslash\{0\}$. Hence $x y-y x=0$, and $x$ is central. Claim 2. If $C \neq \varnothing$, then $C \subset Z(R)$.

Suppose that $x \in C \backslash Z(R)$. Let $y \in R$ be such that $x y \neq y x$. Without loss of generality, we may assume $x>y>0$. By assumption on $R$, we have

$$
\left|x^{3}(x y-y x)\right|=\left|x\left(x^{3} y\right)-\left(x^{3} y\right) x\right| \leq n x^{2} \quad \text { for some } n \in \mathbf{N} \text {. }
$$

Since $D$ is a domain, it follows that $|x(x y-y x)| \leq n$. But as $x>m \forall m \in \mathbf{N}$, we conclude that $0 \neq x y-y x \in A \neq \varnothing$. Thus by Claim 1, both $x y-y x$ and $x(x y-y x)$ are central. Moreover, as $R$ is a domain, $x$ must be central. This is a contradiction.

So if $C=\varnothing$, then we are done by Claim 1. If not, by Claim 2, we have $C \subset Z(R)$. But for any $b \in A \cup B, b+C \subset C \subset Z(R)$. Hence $R$ must be commutative.

In case $R$ contains no identity, as we assume $R$ containis no zero divisors, $(R, P)$ can be order-imbedded in an ordered ring $\left(R^{\prime}, P^{\prime}\right)$ with identity. For reference, see [F, p. 111]. Thus $A, B, C$ are well defined in $R^{\prime}$. The argument above still works if all elements concerned are restricted to be in $R$.

Corollary 4.2. Let $(R, P)$ be an ordered domain. If for any $x, y$ in $R$, there exists $n=n(x, y) \in \mathbf{N}$ such that

$$
x^{2 n}+y^{2 n} \geq 2 x^{n} y^{n},
$$

then $R$ is commutative. In particular, $R$ is commutative if $f\left(x_{1}, x_{2}\right)=$ $x_{1}^{2}-2 x_{1} x_{2}+x_{2}^{2}$ is P.S.D.

Proof. In view of Proposition 4.1, it suffices to show that $\forall x, y \in R$, $\exists m(x, y) \in \mathbf{N}$ such that $|x y-y x|<m(x, y) \min \left\{x^{2}, y^{2}\right\}$. Clearly, we may assume $x>y>0$.

Case (i). $x y>y x$. Thus $x^{i} y>y x^{i}$. By assumption, there exists $n \in \mathbf{N}$ such that

$$
x^{2 n}+(x+y)^{2 n} \geq 2 x^{n}(x+y)^{n} .
$$

After expanding $(x+y)^{2 n}$ and $(x+y)^{n}$, we then move all linear terms with respect to $y$ to the right and all other terms to the left. Next we replace every term on the left by $x^{2 n-2} y^{2}$ and get

$$
M x^{2 n-2} y^{2} \geq x^{2 n-1} y+x^{2 n-2} y x+\cdots+x^{n} y x^{n-1}-x^{n-1} y x^{n}-\cdots-y x^{2 n-1} \geq 0
$$


for some $M \in \mathbf{N}$. But as $x^{r} y x^{2 n-r} \leq x^{n+r} y x^{n-r}$ for all $r \leq n$, so the R.H.S. $\geq$ $\left|x^{2 n-1} y-x^{2 n-2} y x\right|$. Therefore, we have

$$
\left|x^{2 n-1} y-x^{2 n-2} y x\right| \leq M x^{2 n-2} y^{2} \text {. }
$$

Since $R$ is a domain, we can cancel $x^{2 n-2}$ from both sides. It follows that $|x y-y x| \leq M y^{2}$.

Case (ii). $x y<y x$. In this case, we consider the inequality $(x+y)^{2 m}+x^{2 m} \geq$ $2(x+y)^{m} x^{m}$. As before, we get the desired inequality.

Finally, we give an example to show that if $R$ is not a domain, then the above Corollary is not true for $n=1$.

Example. Let $x, y$ be noncommutative indeterminates and $\mathbf{Z}^{\prime}[x, y] \subset \mathbf{Z}[x, y]$ be the subring generated by $x$ and $y$. In $\mathbf{Z}^{\prime}[x, y]$, we define $I:=$ the ideal generated by $\left\{x^{3}, x^{2} y, x y x, x y^{2}, y^{3}, y^{2} x, y x y, y x^{2}\right\}$ and $R:=\mathbf{Z}^{\prime}[x, y] / I$. Obviously,

$$
R=\left\{a_{1} \bar{x}+a_{2} \bar{y}+a_{3} \bar{x}^{2}+a_{4} \overline{x y}+a_{5} \bar{y}^{2}+a_{6} \overline{x y-y x}: a_{i} \in \mathbf{Z} i=1,2, \ldots, 6\right\} .
$$

Note that if $a_{i} \neq 0$ for some $i$, then $a_{1} \bar{x}+a_{2} \bar{y}+a_{3} \bar{x}^{2}+a_{4} \overline{x y}+a_{5} \bar{y}^{2}+a_{6} \overline{x y-y x} \neq$ 0 . For convenience, we shall use the following notation:

$$
\begin{aligned}
& e_{1}:=\bar{x}, e_{2}:=\bar{y}, e_{3}:=\bar{x}^{2}, \\
& e_{4}:=\overline{x y}, e_{5}:=\bar{y}^{2}, e_{6}:=\overline{x y-y x} .
\end{aligned}
$$

Next, let $v: R \rightarrow \mathbf{Z}$ be a mapping such that

$$
v\left(\sum_{i=1}^{6} a_{i} e_{i}\right)= \begin{cases}a_{i} & \text { where } a_{i} \text { is the first nonzero coefficient } \\ 0 & \text { if all } a_{i} \text { are zero }\end{cases}
$$

Furthermore, if $f:=\sum_{i=1}^{6} a_{i} e_{i}$ and $v(f)=a_{j}$, we say the leading term of $f$ is $a_{j} e_{j}$. Now, let us define

$$
P:=\{f \in R: v(f) \geq 0\} .
$$

It is straightforward to show that $P$ is closed under addition and multiplication.

Finally, we show that for any $f, g \in R$,

$$
|f g-g f| \leq \min \left\{f^{2}, g^{2}\right\} \text {. }
$$

Let $f:=\sum_{i=1}^{6} a_{i} e_{i}$ and $g:=\sum_{i=1}^{6} b_{i} e_{i}$ be in $R$. Then by a straightforward calculation, we see that

$$
f g=a_{1} b_{1} \bar{x}^{2}+a_{1} b_{2} \overline{x y}+a_{2} b_{1} \overline{y x}+a_{2} b_{2} \bar{y}^{2}
$$

and

$$
f g-g f=\left(a_{1} b_{2}-a_{2} b_{1}\right) \overline{x y-y x}=\left(a_{1} b_{2}-b_{1} a_{2}\right) e_{6} .
$$

If $f g-g f=0$, then obviously inequality (4) holds. If $f g-g f \neq 0$, then by the calculation above, we see that $a_{1}, b_{1} \neq 0$ and the leading term of $|f g-g f|$ is of the form $b e_{6}$. Clearly, the leading terms of $f^{2}$ and $g^{2}$ are $a_{1}^{2} e_{3}$ and 
$b_{1}^{2} e_{3}$ respectively. Therefore, the leading term of $\min \left\{f^{2}, g^{2}\right\}$ is of the form $a^{2} e_{3}$. Hence, we see that $\min \left\{f^{2}, g^{2}\right\}>|f g-g f|$.

\section{ACKNOWLEDGMENT}

The author would like to thank Professor T. Y. Lam, Professor G. Bergman and Tara Smith for reading the manuscript and giving some valuable suggestions. Also, the author would like to thank the referee for pointing out some mistakes in the first example given in $\S 4$.

\section{REFERENCES}

[A] A. A. Albert, On ordered algebras, Bull. Amer. Math. Soc. 45 (1940) 521-522.

[F] L. Fuchs, Partially ordered algebraic systems, Pergamon Press, Oxford, London, New York, Paris, 1963.

[CL 1 ] M. D. Choi and T. Y. Lam, An old question of Hilbert, in Proceedings of the Quadratic Form Conference 1976, ed. G. Orzech, 385-405. Queen's Papers in Pure and Applied Mathematics, vol. 46, Kingston, Ont., Queen's University.

$\left[\mathrm{CL}_{2}\right]$ - Extremal positive semidefinite forms, Math. Ann. 231 (1977), 1-18.

[CLR 1 ] M. D. Choi, T. Y. Lam and B. Reznick, A combinatorial theory for sums of squares, see Abstract Amer. Math. Soc., 738-12-30.

[CLR ${ }_{2}$ ] _ Symmetric quartic forms, see Abstract Amer. Math. Soc., 736-10-21.

[CLR 3 ] - Even symmetric sextics, Math. Z. 195 (1987), 559-580.

[L] T. Y. Lam, The theory of ordered fields, Ring Theory and Algebra III, ed. B. McDonald, Lectures Notes in Pure and Applied Math., vol. 55, Dekker, New York, 1980, 1-152.

[R] R. M. Robinson, Some definite polynomials which are not sum of squares of real polynomials, Selected Question of Algebra and Logic, 264-282, Acad. Sci. USSR, 1973 (see abstract in Notices Amer. Math. Soc. 16554 (1969)).

Department of Mathematics, National University of Singapore, Singapore, 0511 